\title{
Service militaire et intégration nationale pendant la révolution française
}

\section{Thomas Hippler}

\section{(2) OpenEdition \\ 1 Journals}

\section{Édition électronique}

URL : https://journals.openedition.org/ahrf/662

DOI : 10.4000/ahrf.662

ISSN : 1952-403X

Éditeur :

Armand Colin, Société des études robespierristes

\section{Édition imprimée}

Date de publication : 1 septembre 2002

Pagination : 1-16

ISSN : 0003-4436

\section{Référence électronique}

Thomas Hippler, « Service militaire et intégration nationale pendant la révolution française », Annales historiques de la Révolution française [En ligne], 329 | juillet-septembre 2002, mis en ligne le 27 mars 2008, consulté le 23 avril 2022. URL : http://journals.openedition.org/ahrf/662 ; DOI : https://doi.org/ 10.4000/ahrf.662 


\title{
SERVICE MILITAIRE ET INTÉGRATION NATIONALE PENDANT LA RÉVOLUTION FRANÇAISE (1)
}

\author{
THOMAS HIPPLER
}

\begin{abstract}
L'article analyse le rôle du service militaire dans la formation du lien civique pendant la Révolution. Pour ce faire, le service est appréhendé selon sa double caractéristique d'appareil disciplinaire, d'une part, et, d'autre part, d'exercice de la citoyenneté. Est développé l'argument selon lequel la tension entre droits civiques et sujétion disciplinaire qui se manifeste dans le service militaire s'accompagne d'un rapport conflictuel avec certaines catégories identitaires (de genre, de classe, de nation et de race) dans lesquelles se reconnaît le citoyen et selon lesquelles il est reconnu en tant que tel.
\end{abstract}

Mots clés : Service militaire ; conscription; soldats ; armée ; citoyenneté; discipline.

Le service militaire est une des institutions les plus paradoxales de l'État moderne. Depuis la Révolution française, l'exercice de la violence physique - considéré par Max Weber comme fonction fondamentale de l'État (2) - est assumé par la «nation », c'est-à-dire l'ensemble des citoyens masculins. Dans la définition française de la république, la citoyenneté ne peut pas être séparée d'un certain nombre de devoirs envers l'État, dont le service militaire est probablement un des plus importants. Or, l'individu soumis à la conscription ne jouit pas de la plénitude de seśddroits civiques, ni

(1) Cet article est issu d'une conférence tenue à l'Institut historique allemand à Paris en janvier 2001 et reprend des arguments qui seront développés dans une thèse de doctorat en cours à l'Institut universitaire européen de Florence portant sur la conscription en France et en Prusse entre 1789 et 1830. Je voudrais remercier Valérie Mathevon, Julie Ringelheim et Emmanuelle Rosso pour avoir relu et corrigé le manuscrit ainsi que les professeurs Annie Crépin, Peter Becker, Werner Paravicini, Bo Strath et Peter Wagner pour leur aide et leurs conseils.

(2) Max WEBER, Wirtschaft und Gesellschaft. Grundriß der verstehenden Soziologie, Tübingen, Mohr, 5e éd. 1972 : "Staat ist diejenige menschliche Gemeinschaft, welche innerhalb eines bestimmten Gebiets [...] das Monopol legitimer physischer Gewaltsamkeit für sich (mit Erfolg) beansprucht. " 
même des libertés fondamentales que la conscription est pourtant censée défendre. Appareil disciplinaire d'État d'une part, et, d'autre part, promesse de liberté politique, le service militaire est une des institutions qui reflètent le mieux l'ambiguité fondamentale de la modernité politique (3).

Ainsi, une analyse historique du service militaire pourrait éclairer certains aspects et donner un fondement empirique à un débat qui, pour l'instant, a plutôt occupé les sociologues et les philosophes. Paradoxalement, une des institutions les plus significatives de l'État moderne ne commence à intéresser les historiens que depuis l'annonce de son abolition (4). Or, en étudiant à travers le service militaire la manière dont l'État et les individus qui le constituent en tant que "peuple» ont façonné leurs relations mutuelles, on s'aperçoit que d'autres «constructions identitaires" sont à prendre en compte.

Historiquement, l'État s'est formé comme État national, ainsi les individus soumis au devoir militaire deviennent des citoyens «nationaux». Le service, toutefois, ne concerne pas tous les "nationaux " indistinctement, puisque la participation féminine est exclue d'emblée. De cette manière, l'armée nationale devient un des lieux où la masculinité, et donc les identités sexuelles et leur différence, sont produites et reproduites à l'échelle des institutions. Enfin, face à une tendance constante à «nationaliser » l'armée, des intérêts de classe se manifestent, par le remplacement et l'exemption de catégories professionnelles entières.

Nous allons étudier dans les pages qui suivent certains aspects de l'expérience révolutionnaire concernant l'armement populaire et la façon dont l'État a «récupéré » l'appropriation de la violence légitime par le peuple en armes. Pour ce faire, deux types de sources ont été principalement utilisées : d'une part, des discours à forte charge théorique comme ceux des parlementaires, les écrits des publicistes et les textes philosophiques, d'autre part, des écrits personnels de soldats et de volontaires de la Révolution. La juxtaposition des deux permettra de détecter la corrélation et les interactions entre l'idéologie révolutionnaire et l'expérience vécue par ceux qui ont tout donné pour cette Révolution.

(3) Peter WAGNER, Soziologie der Moderne : Freiheit und Disziplin, Frankfurt-New York, Campus, 1995.

(4) Cf. les études récentes d'Annie CRÉPIN, La conscription en débat ou le triple apprentissage de la nation, de la citoyenneté, de la République (1798-1889), Arras, Artois Presses Université, 1998; Odile ROYNETTE, "Bons pour le service ": l'expérience de la caseme en France à la fin du XIXe siècle, Paris, Belin, 2000; Maurice VAīssE (dir.), Aux armes, citoyens! Conscription et armée de métier des Grecs à nos jours, Paris, Armand Colin, 1998; Frédéric RousSEAU, Service militaire au XIXé siècle : de la résistance à l'obéissance. Un siècle d'apprentissage de la patrie dans le département de l'Hérault, Montpellier, UMR 5609 du CNRS-ESID « États-Sociétés-Idéologies-Défense », 1998. 
L'organisation militaire, ou plus précisément l'organisation de la « force publique ", fut un problèmé politique d'importance majeure dès le commencement de la Révolution. Depuis 1789, des Gardes nationales se formèrent un peu partout en France, mais on ne peut les qualifier de véritable force militaire : elles représentaient plutôt une sorte de police auxiliaire dont l'accès était réservé aux citoyens actifs. Le nom initial de la Garde nationale de Paris, "Milice bourgeoise », rend mieux compte du caractère social de cette force armée. Formée spontanément avec la prise de la Bastille et immédiatement organisée sous le commandement de La Fayette, la Garde nationale doit être considérée comme le Tiers État en armes, par opposition aux troupes royales d'une part, et aux révoltes populaires (les paysans pendant l'été, les Parisiens en juillet et en octobre) de l'autre.

C'est dans de telles conditions que s'ouvre, en décembre 1789, un débat à l'Assemblée nationale concernant l'organisation militaire. L'enjeu relève de la pure politique intérieure et de la définition des rapports entre forces armées et société que l'on s'efforce de préciser; aucune menace de guerre ne pèse sur la France à cette époque. Des cahiers de doléances ainsi que des réclamations de militaires destinées au ministère de la Guerre témoignent du désir largement partagé d'un changement dans l'organisation militaire. Alors que les militaires revendiquaient l'abolition de la milice et des peines dégradantes, un traitement plus «humain » des soldats (5) et l'accès aux droits civiques pour les militaires (6), les députés, quant à eux, souhaitaient donner à la France une constitution militaire qui coïncide mieux avec le partage du pouvoir nouvellement imposé au roi, à savoir un rééquilibrage de la constitution militaire à l'aune des fondements nouveaux de la constitution politique (7).

Il y avait consensus à l'Assemblée sur le fait de rechercher désormais dans la «nation» la base de la nouvelle politique du recrutement militaire selon le principe que «tous les citoyens sont les défenseurs de la patrie». Tous les orateurs considéraient la loi qu'ils allaient voter comme une des principales de tout l'édifice constitutionnel à bâtir : «La formation de l'armée est, dans toute espèce de gouvernement, un des points essentiels de la constitution [...] De toutes les parties qui forment l'ensemble de la constitution militaire, [...] le mode de Recrutement est celui que l'on peut regarder comme la plus essentielle » disait le duc de Liancourt (8). Le recrutement de

(5) S.H.A.T. (Service historique de l'armée de terre), M.R. 1907, p. 4.

(6) Ibid., p. 17.

(7) « Établir un ordre nouveau, étoit le premier de vos devoirs; le second étoit de créer les moyens de maintenir cet ordre, \& de le rendre durable. " RABAUT, Rapport sur l'organisation de la force publique, fait au nom du Comité de Constitution \& du Comité militaire, le 21 novembre 1790, Paris, Imprimerie nationale, 1790 , p. 1.

(8) Liancourt, Opinion sur le mode de recrutement pour l'armée, par M. le Duc de Liancourt, député de Clermont en Beauvoisis, Paris, Imprimerie nationale, décembre 1789, pp. 1-2. 
l'armée est un problème politique de la plus haute importance puisqu'il s'agit de déterminer qui porte les armes au nom de l'État et à quel titre. Mais l'accord entre les partisans d'une obligation militaire universelle et leurs adversaires s'arrêta là.

Le ministre de la Guerre, La Tour du Pin, dans un mémoire exposant son projet de loi relatif au recrutement, reconnaissait que «tous les citoyens se doivent sans doute à la défense de l'État » mais il ajouta qu'une « distinction importante doit être faite dans l'application de cette vérité » (9): la conciliation entre deux principes politiques contradictoires, l'obligation de concourir à la défense du pays d'une part, et la liberté individuelle de l'autre, nécessitait un compromis qui consisterait en une séparation ordonnée des tâches entres «les classes politiques différentes » (10).

Nous touchons là précisément au point central du désaccord : les partisans d'une obligation militaire universelle mettaient en question les divisions de la société en différentes classes et avant tout la séparation entre militaires et civils. Ce genre d'argument était évidemment très litigieux car il tendait aussi à remettre en cause la séparation politique primordiale entre citoyenneté active et citoyenneté passive (11). Dubois-Crancé, le porte-parole des défenseurs de la conscription, soutint qu'une exclusion des citoyens passifs de la Garde nationale entraînerait le relâchement des liens entre les différentes classes et mènerait à une désintégration de la nation (12). Et si, de surcroît, l'armée régulière continuait à recruter exclusivement des volontaires, c'est-à-dire en pratique des gens issus de « la classe la plus indigente, j'ai failli dire la plus vile de la nation ", comme le formulaient Cessac et Servan, alors on continuerait à avoir une armée qu'aucun lien n'attacherait à la nation et qui ne pourrait être domptée que par une discipline de fer (13).

Le différend entre les partisans de la conscription et ses opposants a pour fondement deux conceptions antagonistes de la «liberté politique». Tandis que les uns considèrent le service militaire obligatoire comme le comble du despotisme et la négation absolue de la liberté, les autres défendent l'idée que celle-ci ne se joue pas uniquement au niveau individuel, mais relève d'un problème politique étroitement lié à l'existence d'une "force publique ». Il faut un État fort car, selon la formule de Dubois-Crancé, «la liberté est une chimère, si le plus fort peut impunément opprimer le plus

(9) LA TOUR DU PiN, Mémoire lu par M. le comte de la Tour du Pin, ministre \& secrétaire d'État de la Guerre au Comité nommé par l'Assemblée nationale, Paris, Imprimerie royale, 1789, p. 3.

(10) GOUPY DE MORVILLE, Vues générales d'un plan politique concernant une nouvelle constitution de l'armée nationale française, et un projet de finance relatif, pour l'anéantissement futur de toutes pensions militaires, Paris, Philippe-Denys Pierres, 1790, p. 10.

(11) Albert SOBOUL, Les soldats de l'an II, Paris, Club français du livre, 1959.

(12) DUBOIS DE CRANCÉ, Discours sur la force publique et sur l'organisation des gardes nationales du royaume, Paris 1790 , p. 11.

(13) CESSAC-SERVAN, Projet de constitution pour l'armée des François, présenté au comité militaire de l'Assemblée nationale, Paris, Boudouin, s.d., p. 18. 
foible » (14). Il n'y a de vraie liberté que dans une république; l'absence d'État équivaut à l'oppression du plus faible par le plus fort et conduit ainsi à l'esclavage (15). La résistance à ce genre d'oppression est donc à la fois un " devoir sacré » et un droit inaliénable de chaque citoyen.

Or, pour que la liberté civile existe, la «force publique » doit être subordonnée à la volonté générale qui, selon Rousseau, n'est jamais dans l'erreur (16). C'est pourquoi même ceux qui voulaient imposer la conscription à la France admettaient que sous la tyrannie ce serait «l'excès du despotisme [que d'] être obligé de porter les armes pour s'opprimer soimême » (17). Dans une «nation souveraine ", en revanche, c'est-à-dire dans un État où les lois sont l'expression de la volonté générale, tous les citoyens n'obéissent par définition qu'aux lois dont ils sont les auteurs, puisqu'ils constituent le peuple souverain. Dans la mesure où la souveraineté ne peut pas être déléguée ou représentée, elle doit toujours rester aux mains des citoyens : "la souveraineté n'étant que l'exercice de la volonté générale, ne peut jamais s'aliéner, et [...] le souverain, qui n'est qu'un être collectif, ne peut être représenté que par lui-même " (18). C'est dans ce sens que l'on a pu dire que la conscription est «le palladium des peuples libres» (19). Le peuple est souverain dans l'exacte mesure où la force publique de l'État dans lequel il se constitue reste la sienne.

Dans cette conception, qui reprend un héritage intellectuel ancien allant de Machiavel à Rousseau et Mably en passant par bien d'autres, le service militaire peut être le facteur d'une synthèse nationale qui est susceptible de contribuer à la formation d'un nouveau type de relation politique (20). Sont en jeu des « sujets dociles » pour qui la loi sera « connue, $\&$ par conséquent respectée \& chérie ", comme le disait Rabaut dans un rapport à l'Assemblée nationale l'année suivante. Le soldat-citoyen sera formé par les «fêtes militaires, les fêtes politiques, les institutions civiles d'éducation nationale, qui, dans un même temps \& sous les mêmes formes, transmettent à tous les citoyens les mêmes sentiments, les mêmes usages, les mêmes mœurs » et aideront à développer «cette vénération religieuse, avec

(14) DUbois de CRANCÉ, Discours... op. cit., p. 9.

(15) Selon Rousseau il faut « distinguer la liberté naturelle, qui n'a pour bornes que les forces de l'individu, de la liberté civile, qui est limitée par la volonté générale [...] la liberté morale, qui seule rend l'homme vraiment maître de lui; car l'impulsion du seul appétit est esclavage, et l'obéissance à la loi qu'on' s'est prescrite est liberté », Jean-Jacques RousSEAU, « Du contrat social ou principes du droit politique " dans Euvres complètes, tome 2, Paris, Seuil (coll. L'Intégrale), 1971, p. 524.

(16) « La volonté générale est toujours droite et tend toujours à l'utilité publique », ibid., p. 527.

(17) Article "Conscription militaire » dans Encyclopédie méthodique..., Tome Quatrième (supplément) Art militaire, Paris, chez H. Agasse, M. DCC. XCVII, L'an V de la République françoise, p. 199.

(18) Rousseau, Du Contrat social... op. cit., p. 525.

(19) KERSAINT, Considérations sur la force publique, et l'institution constitutionnelle des gardes nationales... Paris, Imprimerie du patriote français, mai 1791, p. 7.

(20) Cf. à ce sujet R. Claire SNYDER, Citizen-Soldiers and Manly Warriors : Military Senvice and Gender in the Civic Republic Tradition, Lanham, Rowman \& Littlefield, 1999. 
laquelle le citoyen incline son front devant la sainte autorité de la loi, cet amour de la constitution qui s'identifie avec l'amour du pays » (21).

L'individu devient citoyen dans l'exacte mesure où il devient cette matière dans laquelle les normes universelles de liberté et d'égalité seront imprimées; il est conçu comme pars totalis de la communauté politique, véritable synthèse du particulier et de l'universel. Cependant, nous sommes en 1789-1790 et pas encore en 1793. Liancourt, le plus éloquent des adversaires de la conscription, s'écrie que « l'habitude de la Constitution nouvelle attachant, par la réflexion, par le bonheur, les citoyens à tous les intérêts de l'État, peut seule, aidée du temps, agir sur les opinions, changer les mœurs, \& amener dans les esprits cette grande révolution par laquelle, seulement, une direction nouvelle peut être donnée aux habitudes \& aux idées. Mais, en attendant qu'elle s'opère, les citoyens seront esclaves, \& le service de l'Armée fait mal » (22). L'argument est que, même si le but poursuivi par l'instauration d'un service militaire est louable en soi, le moyen d'y parvenir ressemble fâcheusement à une dictature éducatrice. La formation de la grande synthèse nationale doit être le fruit du temps et non de la contrainte. Liancourt parvient à rallier la majorité des députés à ses vues et la nouvelle loi stipule que les troupes françaises se recrutent par engagements volontaires et non par conscription.

\section{II}

Cette législation de 1789 fut érodée progressivement par l'évolution de la Révolution. La désintégration de l'armée royale, la déclaration de guerre et la radicalisation politique furent les facteurs principaux de l'extension des droits et des devoirs militaires à l'ensemble de la population masculine du pays (23).

Dès 1791, avec la menace de guerre qui pesait sur la France, et surtout après la fuite du roi en juin, on décida de lever des bataillons de volontaires parmi la Garde nationale; socialement, ces «volontaires de 1791 » sont majoritairement issus de la petite bourgeoisie (à Paris, $90 \%$ sont artisans ou boutiquiers), les officiers, en revanche, sont souvent des nobles. Il importe de souligner que les bataillons de volontaires étaient organisés séparément de l'armée régulière : les volontaires portaient un autre uniforme, étaient payés différemment, élisaient leurs officiers et n'étaient pas soumis aux

(21) RABAUT, Rapport... op. cit., pp. $2-8$.

(22) LIANCOURT, Opinion (...), op. cit., p. 8.

(23) Jean-Paul BERTAUD, La Révolution amée : Les soldats-citoyens et la Révolution française, Paris, Robert Laffont, 1979. Pour les paragraphes qui suivent, on se rapportera essentiellement à ce livre qui reste incontournable pour tout ce qui touche à l'armée de la Révolution. 
contraintes habituelles de l'état militaire comme par exemple l'interdiction du mariage. Après la déclaration de guerre en avril 1792, on leva des légions et des compagnies franches, théoriquement par volontariat, mais on essaya d'attirer des anciens militaires; on incorpora également les anciens régiments étrangers, et on fit appel de nouveau aux volontaires. L'Assemblée nationale leva ensuite des corps de "fédérés" parmi les révolutionnaires (décret du 8 juin 1792) qui jouèrent un rôle important dans les journées du 20 juin et du 10 août. Le 24 février 1793, la Convention décréta la levée de 300000 hommes qui ressemble à certains égards à la conscription : tous les hommes âgés de 18 à 40 ans, célibataires ou veufs sans enfants, étaient en état de réquisition permanente. Pourtant, comme le souligne Jean-Paul Bertaud, «le service militaire obligatoire pour tous n'était pas institué. Les individus n'étaient concernés qu'à l'intérieur de leur communauté. Or chaque communauté n'avait pas à fournir le même contingent » (24).

Ce processus conduit en quelques mois à un décret sur la «levée en masse » d'août 1793 signalant que « tout Français est soldat », termes repris dans la constitution de la même année. Entre la levée de 300000 hommes en février - qui provoqua le début de la guerre civile dans les départements de l'Ouest - et la levée en masse en juillet, un changement essentiel s'opère : dès lors, l'obligation militaire n'est plus définie en fonction de l'appartenance communale - le gouvernement faisant appel aux départements et cantons pour qu'ils fournissent un certain nombre de soldats - mais découle directement de la qualité même de citoyen. Le service militaire instaure donc une relation d'appartenance et de service personnel entre l'individu et l'État.

Quant aux modalités de la levée en masse, notons les divisions à l'intérieur même du parti révolutionnaire, en particulier entre les sans-culottes et le gouvernement jacobin. L'idée d'une levée en masse émergea en effet dans les sections des sans-culottes parisiens au printemps 1793 et s'inséra dans tout un réseau de revendications concernant le contrôle politique de l'économie, la fixation des prix, la régénération nationale par l'extermination des rebelles, et une sorte d'institutionnalisation du droit à l'insurrection populaire (25). Pour les sans-culottes, il se s'agissait pas d'inventer un nouveau mode de recrutement de l'armée. Leur rhétorique est plutôt celle de l'ultime combat pour l'humanité, d'un déploiement gigantesque de toutes les forces vives du peuple destinées à porter un coup meurtrier aux divisions politiques, à la trahison, à la domination bourgeoise et à l'oppression. "Huit jours d'enthousiasme peuvent faire plus pour la patrie que huit ans de

(24) Id., p. 100.

(25) Albert SoBouL, Les sans-culottes parisiens en l'an II: Mouvement populaire et gouvemement révolutionnaire, 2 juin 1793 - 9 thermidor an II, Paris, Clavreuil. 1958, p. 109. 
combat ", disait Sébastien Lacroix à la section de l'Unité le 28 juillet (26). Évidemment, le gouvernement, et Robespierre en particulier, n'était guère enclin à approuver de telles mesures. Ainsi, le décret sur l'exécution de la levée en masse du 23 août différa sensiblement des intentions des sansculottes, et ce d'autant plus que les formes de démocratie directe en vigueur dans les bataillons de volontaires nationaux étaient en voie de disparition. L'action des commissaires jacobins aux armées, l'«amalgame" des bataillons de volontaires avec les troupes de ligne et l'application d'un code disciplinaire plus strict contribuèrent à éteindre la démocratie directe dans les armées de la Révolution (27).

La ligne de démarcation entre, d'une part, les sans-culottes et l'armement « anarchique » du peuple qu'ils revendiquent et, d'autre part la «politique de la vertu » des Jacobins, concerne donc l'institutionnalisation du cadre étatique propre à contenir - dans les deux sens du mot - la force publique. Ce qui est en jeu est la définition de la souveraineté populaire. Le «peuple » est source de toute souveraineté parce qu'il est source de la force publique; mais ce même «peuple » est aussi un facteur permanent de déstabilisation sociale. Si les sans-culottes radicaux considèrent la souveraineté populaire comme résidant essentiellement dans le droit à l'insurrection permanente (28), les Jacobins soutiennent qu'un «peuple» n'est pas une entité naturellement donnée mais doit se constituer par l'action permanente de la vertu. Une masse farouche en insurrection n'est et ne peut pas être un peuple puisqu'elle est habitée par le vice : d'où la nécessité d'institutions disciplinaires pour soutenir le développement de la vertu en particulier dans un régime démocratique. La discipline extirpe le vice et constitue le peuple en tant que tel.

De manière significative, vertu et vice sont considérés comme des catégories sexuées : la vertu est masculine et le vice féminin. La présence des femmes dans la société est donc une menace perpétuelle pour l'établissement d'un ordre moral, ce qui nécessite qu'elles soient contenues dans des limites étroites. Comme le dit Mably «Il n'en faut pas davantage pour subjuguer le plus brave homme; et si nous sommes domptés, vous n'aurez qu'une république de femmelettes » (29). Davantage : le vice est antisocial par définition, puisqu'il consiste à faire prévaloir l'intérêt particulier; la vertu, en revanche, est une catégorie centrale de sociabilité portée vers l'intérêt

(26) Sébastien LACROIX, Pas un moment à perdre ou Discours prononcé par le citoyen Lacroix à la section de l'Unité, dans la séance du 28 juillet [1793], Paris, Guyot, s.d.

(27) Jean-Paul BERTAUD, La Révolution armée ..., op. cit., p. 84.

(28) «L'ultime recours du peuple souverain, c'est l'insurrection. [...] Les sans-culottes ne virent pas là une affirmation théorique et formelle de leur souveraineté.", Albert SoBOuL, Les sans-culottes.., op. cit., p. 542 .

(29) MABLY, « De la législation, ou principes des lois » dans Collection complète des auvres de l'abbé de Mably, tome 9, Paris, Desbriere, l'an III de la République (1794 à 1795), p. 376. 
général. La « politique de la vertu » doit, par conséquent, être sexuée aussi ; ainsi il est nécessaire de circonscrire l'influence féminine et, corrélativement, de renforcer la masculinité de l'homme, par le biais notamment des exercices militaires. En effet ces derniers inspirent le sens de la "gloire " qui représente le moyen idéal pour que l'individu s'affranchisse de ses penchants vicieux et devienne entièrement vertueux. La gloire «semble en quelque sorte nous séparer de nous-mêmes, nous nous oublions par une sorte de prestige; prêts à lui sacrifier notre vie, l'image d'une belle mort s'empare de notre âme et l'enivre » (30).

Le citoyen-soldat est en "extase " : il est plus qu'un simple individu, puisqu'il est dépouillé de toute identité et de tout intérêt particulier et ainsi de tout vice. L'armée, où «les héros de la liberté s'endurcissent aux fatigues et apprennent à braver la mort pour assurer le triomphe de la République », est le lieu par excellence où cette nouvelle subjectivité civique est censée se former : "La mort est un rappel à l'égalité, qu'un peuple libre doit consacrer par un acte public qui lui retrace sans cesse cet avertissement nécessaire » (31).

Mais cette égalité dans la mort est aussi un universel bien particulier qui ne concerne qu'une partie déterminée de l'humanité : dans la mesure même où le caractère de classe s'efface progressivement devant le droit et l'obligation de porter les armes, la nationalisation et l'affirmation du caractère masculin de.la force armée progressent. Dans les discours officiels, il n'a jamais été question d'un élargissement des droits civiques aux femmes, et encore moins de leur permettre de porter les armes (32). D'une manière très significative, le mouvement des «citoyennes républicaines révolutionnaires " qui s'est constitué dès mars 1792 avait comme but premier de former une "garde nationale féminine" (33) revendiquant ainsi un rôle politique actif pour les femmes (34). Un mouvement comparable peut être observé dans les bataillons de volontaires des armées de la Révolution : bien que les appels aux volontaires nationaux n'aient été adressés qu'aux hommes, un certain nombre de femmes s'enrôlèrent, la plupart déguisées en

(30) MABLY, « Entretiens de Phocion, sur le rapport de la morale avec la politique. » dans Collection complète des ceuvres de l'abbé de Mably, op. cit., tome 10, p. 126.

(31) BILlaUd-VARENNE, Rapport fait à la Convention nationale... dans la séance du premier floréal, l'an 2 «Sur la théorie du gouvernement démocratique, et sa vigueur utile pour contenir l'ambition, et pour tempérer l'essor de l'esprit militaire; sur le but politique de la guerre actuelle; et sur la nécessité d'inspirer l'amour des vertus civiles par des fêtes publiques et des institutions morales », Paris, Imprimerie nationale, an II, pp. 19- 24.

(32) Dominique Godineau, Citoyennes tricoteuses: les femmes du peuple à Paris pendant la Révolution française, Aix-en-Provence, Aliéna, 1988, p. 119.

(33) Claude Gulloon, Deux enragés de la Révolution : Leclerc de Lyon et Pauline Léon, Quimperlé, La Digitale, 1993 avec, en annexe, le règlement de la Société des citoyennes républicaines révolutionnaires, pp. 246-49.

(34) Pauline LÉOn, Adresse individuelle à l'Assemblée nationale, par des citoyennes de la capitale, le 6 mars 1792, imprimée par ordre de l'Assemblée nationale, Paris, Imprimerie nationale. s.d. 
hommes (35). Malgré la faiblesse des sources, leur présence dans l'armée est attestée même au-delà du mois d'avril 1793 , date d'un décret expulsant formellement les femmes (à l'exception des blanchisseuses, etc.) de l'armée (36). La justification en était précisément que leur présence menaçait le bon ordre et la discipline.

La nationalisation de l'armée progressa dans le même sens mais moins rapidement : les licenciements des régiments étrangers et les pressions exercées sur des officiers d'origine étrangère afin qu'ils démissionnent (37), furent les premiers signes d'un basculement de l'acception du mot «national » (38). Si, au début de la Révolution, le terme de «nation » a un caractère républicain, il se définit de plus en plus par opposition à d'autres «nations » : l'ethnos se substitue au demos (39).

La loi Jourdan de fructidor an VI (1798) peut être considérée comme l'institutionnalisation de l'expérience révolutionnaire. Elle instaure en principe une obligation militaire personnelle pour l'ensemble de la population mâle du pays mais affirme aussitôt que la prise en compte des réalités économiques et sociales primera sur l'universalité du devoir : «Beaucoup seront destinés à servir mais, vraisemblablement, peu serviront réellement» affirmait-on (40). Selon Annie Crépin, cette « loi charnière [...] tente d'inscrire les intuitions de l'ère révolutionnaire dans la réalité et la durée, en leur imprimant un caractère de régularité. Loi fondatrice, elle inspire très largement les lois militaires ultérieures jusqu'à celle de 1872, et, dans l'immédiat, elle fait partie de l'héritage que Bonaparte recueille de la Révolution » (41).

(35) Jean-Paul BERTAUD, La vie quotidienne des soldats de la Révolution. 1789-1799, Paris, Hachette, 1985, pp. 153 sqq.

(36) Dominique GodineaU, Citoyennes tricoteuses..., op. cit., p. 240.

(37) Cf. la lettre de Haacke, colonel de l'armée française d'origine allemande : «Daignez vous rappeler, mon général, les raisons que j'ai eu l'honneur de vous donner dans un mémoire, qui m'empêchent de me charger du commandement d'un régiment français dont aucun, dans les circonstances présentes, ne voudrait être commandé par un Allemand. J'accepte donc, sans balancer, la place d'adjudant général colonel, et mon application, jointe à l'expérience de mes longs services, m'en rendra bientôt les fonctions faciles. ", cité par Arthur CHUQueT, Lettres de 1792, Paris, Honoré Champion, 1911, p. 5.

(38) S.H.A.T., MR 2018/24 (Supplément Préval) : « Projet pour la nationalisation et l'organisation de l'armée de la République français par le citoyen Albert Romé [1793] ».

(39) Cf. aussi la pétition de Oshée à la Convention nationale : «J'ai été suspendu par Bouchotte, sous prétexte que je suis étranger. [...] Est-il bien vrai d'abord que résidant sans interruption en France depuis 42 ans au service de cette nation, je doive être considéré comme étranger ? " OSHÉE, Pétition du citoyen Robert-Richard Oshée, chef de la 94 e demi-brigade, ex-commandant militaire à Libre-ville, à la convention nationale, Paris, Imprimerie de Vinsard, vendémiaire an III, pp. 1- 3.

(40) JouRdan, Rapport fait par Jourdan (de la Haute-Vienne) au nom de la commission militaire, Sur le recrutement de l'armée de terre, Corps législatif, Conseil des Cinq-Cents. Séance du 2 thermidor an VI. Paris, Imprimerie nationale, Thermidor an VI, p. 6.

(41) Annie CRÉPIN, La conscription..., op. cit., pp. 29-30. 
Dans quelle mesure ces discours reflètent-ils un point de vue communément partagé par ces acteurs historiques que sont les soldats de la Révolution? Leur expérience vécue des événements de la Révolution française correspond-elle aux propos «théoriques" des parlementaires, des publicistes, des philosophes? Tâchons d'approcher ces questions par un examen des sources personnelles provenant des soldats de la Révolution, telles que leurs lettres et leurs cahiers de route.

Les volontaires eux-mêmes avaient un sens aigu de leur qualité de citoyen-soldat. Peu d'entre eux auraient daigné s'enrôler comme soldats quelques années auparavant et pourtant leurs récits des scènes d'incorporation sont pleins d'enthousiasme. Ainsi, on aperçoit à la fois une prise de conscience de l'importance de leur mission militaire et civique, et une nette distanciation envers l'armée : Bricard, volontaire parisien de 1792, raconte que beaucoup de volontaires refusaient de toucher aux équipements qui leur étaient fournis par l'armée, par peur d'être considérés comme soldats (42). Ils préféraient rester volontaires plutôt que soldats, c'est-à-dire une force principalement civile qui combat momentanément pour la liberté et qui n'a que peu de choses à voir avec l'ancienne armée - ce qui correspond d'ailleurs à la réalité, puisque les volontaires, dans un premier temps, ont des bataillons qui leur sont propres. De ce point de vue, l'amalgame des troupes de ligne avec les bataillons des volontaires ne fut pas bien vu de tout le monde et même jugé «strictement impossible» par certains (43). «Faudra-t-il que la France, croyant avoir recouvré la liberté, ait encore besoin pour trouver des défenseurs de recourir à l'impôt odieux et barbare de la milice et des engagements forcés? » (44). Cela souligne bien le caractère «extraordinaire», non seulement complètement novateur mais aussi limité dans le temps, que les bataillons de volontaires avaient aux yeux de la plupart des individus concernés; servir comme volontaire de la Révolution ne signifiait pas ipso facto être partisan de la conscription.

Le citoyen-soldat se considérait avant tout comme citoyen plutôt que comme soldat. Aussi, il était naturellement porté à une grande méfiance envers la discipline militaire et tout ce qu'elle comportait d'inhumain, de dégradant, et d'abrutissant; le principe de l'élection des officiers s'imposa comme une évidence et ne fut généralement pas mis en question. Or, on rencontre des différences significatives dans les critères requis pour être un bon officier et des appréciations diverses sur les candidats élus. Bial, futur

(42) BRICARD, Joumal du canonnier Bricard 1792-1802, Paris, Delagrave, 1891, p. 8.

(43) Sur l'amalgame, cf. Jean-Paul BERTAUD, La révolution armée..., pp. 92-99.

(44) Gabriel NoËL, Au temps des volontaires 1792 : Lettres d'un volontaire de 1792. Présentées et annotées par G. Noël, Paris, Plon, 1912, p. 63. 
colonel de l'armée impériale enrôlé comme volontaire en 1792 à l'âge de 19 ans dans le Bas-Limousin, donne une description très vivante de l'élan patriotique à cette époque et relate les discours faits à cette occasion par des responsables politiques qui émettent le vœu que «tous les grades [soient] attribués naturellement aux jeunes gens instruits et de familles aisées, seuls capables de commander ». Lui-même dit ne pas avoir partagé cette opinion, puisque, selon lui, il faut aussi «du zèle, du dévouement, l'amour de la liberté, enfin le désir de sacrifier sa vie au salut de la Patrie » et qu' " au reste il fallait laisser à la masse le soin d'apprécier le mérite de chacun » (45).

Jean-François Godard, un autre volontaire de 1792, ne partage pas cet avis : «Les officiers, chefs de bataillon, capitaines et lieutenants furent nommés par ces bandes, lesquelles choisissaient ceux qui avait le plus de langue et de soldatesque " (46). Sont élus non pas ceux qui sont socialement les plus dignes, mais au contraire les guerriers les plus farouches. La hiérarchie militaire devient autonome par rapport à l'ordre social tandis qu'un ordre militaire directement calqué sur l'ordre civil aurait offert l'inestimable avantage de maintenir la force armée dans cet état de «civilisation » que la « révolution disciplinaire » du XVIIIe siècle avait introduit dans l'armée (47). Ainsi, les récits des guerres révolutionnaires sont remplis d'exemples de pillage, vols, viols, massacres de civils, etc., normalement attribués aux «brigands » qui se sont glissés dans les armées (48).

De cette manière, les rapports entre les volontaires et la population civile deviennent de plus en plus difficiles. Dans un premier temps, le soldatcitoyen est un défenseur de la liberté et des Droits de l'homme et salué comme tel par la population (49). Or, les choses se passent autrement une fois la guerre commencée. En témoigne le récit d'un enrôlé de 1792, originaire de Versailles, racontant que les habitants d'Aix-la-Chapelle tirèrent sur les Français et eurent même «l'inhumanité » de jeter des blessés par la fenêtre, ce qui démontre l'irritation des volontaires devant des populations hostiles à leur égard.

Les volontaires sont ceux qui ont le plus donné à la nation, aussi ont-ils tendance à se considérer comme sa plus pure incarnation : «Pauvre France,

(45) Bial (colonel), Mémoires ou Souvenirs des Guerres de la Révolution et de l'Empire (1792-1814), publiés d'après le Manuscrit original par Gabriel Soulié. Brive, Lachaise, 1929, p. 36.

(46) Aventures de guerre 1792-1809. Souvenirs et récits de soldats recueillis et publiés par Frédéric Masson, Paris, Boussod, Valadon \& Cie, 1894, p. 3.

(47) André CORVISIER, L'armée française de la fin du XVIe siècle au ministère de Choiseul : le soldat, Paris, PUF, 1964, pp. 129-143.

(48) JANNIN, « Lettres de J.-C. Jannin, sergent-major vaguemestre au premier bataillon de la HauteSaône, 1793 m Avec notes de Paul BuONNEAu dans Camet de la Sabretache VII (1899), pp. 400-414, 449473 ; ici p. 469.

(49) MARQUANT, Camet d'étapes du dragon Marquant: Démarches et actions de l'armée du centre pendant la campagne de 1792, publié d'après le manuscrit original par G. VALLÉE et G. PARISET, ParisNancy, Berger-Levrault, 1898, p. 14. 
que je te plains! Si tous les hommes me ressemblaient, tu serais heureuse. Si les forces de l'intérieur ne nous secondent pas, la France est anéantie; la guerre civile est allumée et le royaume le plus florissant est divisé [...] Je tiendrai malgré toutes les trahisons, jusqu'à la mort; je n'aurai rien à me reprocher. Je fais la guerre pour ma patrie, pour mes parents et pour le peuple qui gémissait dans les fers » (50). Leur qualité de défenseur de la patrie les met, en effet, très vite dans une position d'" avant-garde de la Révolution ", qui a tendance à les séparer de ceux qui suivent avec plus ou moins d'enthousiasme (51). Pour les citoyens-soldats, l'armée devient une sorte d'essence de la patrie et l'expérience dominante qu'ils ont pu en faire. Leurs écrits mettent en évidence que les discours sur l'esprit de sacrifice qu'on trouve dans les propos politiques de l'époque ne sont aucunement des paróles creuses, mais qu'ils correspondent bien à un sentiment largement partagé par la population armée. Les expressions «c'est un sacrifice que la liberté nous impose », « un sacrifice que la Patrie demande », etc., reviennent sans cesse.

Le service qu'ils accomplissent devient ainsi le modèle d'une société parfaite où les intérêts particuliers sont en voie de disparition devant la droiture, le patriotisme, l'abnégation de soi, et l'obéissance. Or, la question de l'obéissance montre très clairement les ambiguités auxquelles les citoyenssoldats sont confrontés : d'une part, les rappels à l'obéissance ne manquent pas. Les volontaires assurent dans leurs lettres qu'ils sont bons soldats, disciplinés et obéissants ; certains vont jusqu'à réclamer des guillotines dans l'armée pour punir les ennemis de l'ordre (52) car il ne saurait y avoir de vraie liberté sans ordre, "l'anarchie " est assimilée à l'esclavage (53). D'autre part, ils se considèrent comme des citoyens libres qui ne se battent que momentanément et volontairement pour le salut de la République (54).

Ainsi «le peuple» et la déstabilisation sociale qui l'accompagne font horreur : «Toute la populace s'y est portée et ont exercé le plus affreux brigandage. [...] Les femmes ressemblaient à des furies; il semble que la force leur était donnée. [...] C'était le spectacle de l'horreur. Il me semblait voir la guerre civile » (55). Il est tout à fait significatif - et ce n'est pas le seul

(50) Pierre Girardon, Lettres de Pierre Girardon, Officier barsuraubois, pendant les guerres de la Révolution (1791-1799), publiées et annotées par Louis MORIN, Bar-sur-Aube, Lebois, 1898, p. 24 (lettre du 6 septembre 1792).

(51) « Vous me dites que mon frère me demande de mes nouvelles et que je lui envoie mon adresse. Il se moque de moi; il croit que c'est comme lui sans doute qui est dans sa chambre à caresser sa femme. Triple bombe! S'il a envie de m'écrire il peut m'écrire quand il voudra, au champ de bataille, au camp d'honneur; à Modane, près le Mont Cenis : voilà mon adresse. Et s'il ignore que ça n'est pas, il peut prendre un fusil et qu'il vienne ", Cent lettres de volontaires de l'an II, éd. René BouSCAYroL, Paris, Aux amateurs de livres, 1987, p. 157.

(52) GIRARDON, op. cit., p. 43.

(53) NoÉL, op. cit., p. 236.

(54) S.H.A.T., T 560, « Papiers Dumez », p. 67.

(55) GIRARDON, op. cit., p. 18 (lettre du 18 février 1792). 
exemple - que les femmes fassent ici leur réapparition : la femme furie, incarnation du vice, du désordre, métonymie de la "populace ", est un topos qui ne se trouve pas seulement dans les écrits philosophiques. En plus des armes, il faut de l'organisation, de la subordination, de la discipline pour transformer la "populace" en "peuple". L'organisation de la garde nationale à Collonges en Bas-Limousin, en 1789 est un bon exemple de cette transformation : «Cette réunion d'hommes sans ordre ni discipline présentait un tableau fort curieux. [...] En effet, une compagnie de 24 à 30 hommes fut de suite organisée sous commandement de Monsieur Boutang. [...] C'était un spectacle bien singulier que la transformation soudaine de ces agriculteurs en soldats disciplinés. Dès ce moment-là, on vit naître dans des masses de la nation cet esprit guerrier et patriotique qui devrait engendrer de si grandes choses" (56). Or, malheureusement, afin d'engendrer de si grandes choses, et pour empêcher que la liberté ne déraille vers l'anarchie, il est nécessaire de lui imposer des limites qui ressemblent fâcheusement à son contraire : « Jadis au sein de l'esclavage / J'avais du moins la liberté ; / Libre aujourd'hui par mon courage / Je suis dans la captivité! » rime FrançoisJoseph Dumez lorsqu'il écrit à sa famille (57).

Le doute ne cesse de grandir à propos de l'idée selon laquelle l'armée serait le «lieu » de la liberté et de l'égalité. En février 1792, Gabriel Noël espère encore, tout en se plaignant que cet espoir ne soit pas partagé par tout le monde : "Un grand nombre de villageois qui sont parmi nous ne désirent que retourner chez eux pour cultiver leurs terres : il y en a qui donnent leur démission. Ils ne voient pas, les insensés, que pour cultiver la terre avec tranquillité et profit, il faut que la patrie n'ait point de risques à courir; leur petit intérêt les aveugle. Et ce sont pourtant les gens de la campagne qui gagnent le plus aux bienfaits de la Constitution" (58). Ils ont bien à y gagner, mais cela ne les empêche pas de penser que les exigences militaires qui vont tellement à l'encontre des besoins de la société civile ne relèvent pas du domaine de la «liberté ». On voit ici «en acte» la dialectique qui était à l'œuvre dans les discours cités précédemment et qui consiste en un basculement de la grande synthèse nationale et militaire dans une sorte de particularisme social. Conçue comme facteur et reflet de l'unité nationale, l'armée ne cesse de produire des mécanismes qui sont inconciliables avec les besoins des autres sphères sociales.

La régénération, autre notion fétiche de la Révolution, en est un bon exemple. L'expérience militaire contribue clairement à la purification de la nation et ceci de plusieurs manières. Purification des «intérêts particuliers » dans le sacrifice pour la nation d'abord; ensuite purification de la nation de

(56) BIAL, op. cit., pp. 28-29.

(57) S.H.A.T., T 560, « Papiers Dumez », p. 85.

(58) NOËL, op. cit., pp. $62-63$ (Lettre du 6 février 1792). 
ses ennemis. Des aristocrates d'une part, "car ils sont indignes de vivre avec des frères et respirer un air sain qu'ils pourraient empoisonner » (59), d'autre part, des brigands, des traitres, des scélérats, des satellites des despotes, des esclaves : « Je vais me porter bien dans ce pays-là [la Vendée] pour purger la terre de ces scélérats qui l'infectent [...] Maintenant je vais tuer ou faire tuer des hommes » (60). « Purger la terre », « exterminer les brigands », « égorger beaucoup de monde", sont des expressions qui reviennent sans cesse, particulièrement dans les descriptions de la guerre civile. Ainsi Marquant, volontaire de 1792: « Nous revînmes gîter dans Virton et Saint-Mard, dont les habitants avaient abandonné leurs maisons et fui dans les bois avec leurs bestiaux. Ils furent pillés. On accusera peut-être les Français qui combattaient pour les Droits de l'homme, d'avoir imité le brigandage de leurs ennemis. Mais ils n'agirent pas ainsi sans raisons. Les habitants de ces deux endroits étaient coupables à notre égard : 1) d'avoir suivi leurs armées en France et de s'y être enrichis des dépouilles de nos malheureux paysans; 2) d'avoir, durant le combat, tiré sur nous [...] 3) parce qu'en fuyant, nous crûmes qu'ils nous regardaient comme des scélérats » (61).

Sans nul doute, de semblables expériences ne manquent pas de modifier les personnalités. «Depuis mon départ du Limousin, une transformation complète s'était opérée en moi et j'avais acquis en ces quinze mois une certaine maturité d'esprit qui surprenait mes camarades " (62) disait l'un; un autre espère plutôt que l'expérience du combat ne nuira pas à son «humanité » : «Le jeu sera cruel et périlleux peut-être mais il n'y faut pas songer. Le soldat combattra avec acharnement sitôt qu'il aura occasion de le faire, et bien qu'ayant appris le terrible métier de tuer, il vous reviendra toujours doux et humain » (63) - on est en janvier 1792, donc avant que la guerre n'éclate. Un autre encore éprouva «une pénible sensation » voyant pour la première fois des morts et des blessés : «Mais j'en vis bientôt un si grand nombre que je m'y habituai et ma sensibilité fut cuirassée pour bien longtemps [...] Heureusement j'en fus quitte pour avoir mon habit tout emplâtré de la cervelle d'un officier qui fut tué à quelques pas devant moi » (64).

Il est difficile de conclure, à ce stade de l'analyse, sur le fonctionnement de la formation du lien politique du soldat de la Révolution mais nous pouvons souligner certains points. Les discours, que ce soit à l'assemblée ou dans les clubs, dans des brochures ou dans les textes philosophiques, sont

(59) GIRARDON, op. cit., p. 15 (lettre du 6 novembre 1791).

(60) Id., p. 22.

(61) MARQUANT, op. cit., p. 183.

(62) NoËL, op. cit., p. 49.

(63) BIAL, op. cit., p. 76.

(64) Philippe-René Girault, Mes campagnes sous la République et l'Empire 1791-1810, La Rochelle, Typographe A. Siret, 1884, p. 5. 
accompagnés d'une expérience vécue par les volontaires de la Révolution et cette expérience rencontre les mêmes apories et les mêmes retournements dialectiques, principalement en ce qui concerne la possibilité de concevoir la «souveraineté populaire", puisque celle-ci se trouve à la fois "dans" le peuple et nécessairement « en retrait » par rapport à lui. Elle est dispersée dans le corps social entier et «une et indivisible » en même temps. C'est pourquoi elle nécessite précisément l'unification de ce corps social et la réduction des différences qui se font continuellement sentir mais cette tentative échoue à chaque fois devant les incompatibilités « identitaires » qui persistent obstinément.

La démocratie consiste en une participation politique active des individus et le combat physique pour la communauté est certainement la forme la plus extrême de toute participation possible. Mais ceux qui sont armés pour l'État doivent être autre chose que des simples individus armés : il est nécessaire qu'ils deviennent des "citoyens ", c'est-à-dire que leur intérêt individuel s'efface devant l'intérêt collectif. S'il en était autrement, la force armée se constituerait en un ou plusieurs corps séparés de la société, ce qui conduirait soit au despotisme, soit à la guerre civile. Or, les individus vivants sont irréductibles à une pure incarnation de l'universalité abstraite de l'État républicain - si ce n'est dans la «mort égalitaire » dont parlait BillaudVarenne. À l'évidence, la pratique révolutionnaire n'est pas aussi «pure» que ces théories et l'idéal d'une citoyenneté universelle peut constamment entrer en contradiction avec des identités particulières. En même temps, les catégories identitaires, d'une part, «investissent » l'universel, en le définissant selon des modalités spécifiques; il est indéniable, d'autre part, que les normes de l'universel agissent à leur tour sur la formation des identités. L'universel civique est «situé » et se définit par rapport aux classes sociales, aux identités sexuelles, à l'ethnicité et à la nationalité; dans le même mouvement, les catégories identitaires comme classe, nation et genre se définissent aussi par rapport à cette universalité qui est représentée par l'État révolutionnaire. Ainsi, ni la conception de l'universel politique, ni les identités qui lui font obstacle ne sont des entités fixes et immuables, mais elles se constituent mutuellement dans leur rapport conflictuel.

Thomas HIPPLER Institut Universitaire Européen Département d'histoire et civilisation

Villa Schifanoia

Via Boccacio, 121

I - 50133 Florence thomas.hippler@iue.it 